December 2011

\title{
\#Socialtagging: Defining its Role in the Academic Library
}

Annalise Ammer

annaliseammer@gmail.com

Katherine Bertel

SUNY Buffalo, ksbertel@buffalo.edu

Follow this and additional works at: https://scholarworks.sjsu.edu/ischoolsrj

Part of the Library and Information Science Commons

\section{Acknowledgements}

The authors would like to thank their professors, Valerie Nesset and Brenda L. Battleson, of the Department of Library and Information Studies at the University at Buffalo, The State University of New York, for their guidance and support.

\section{Recommended Citation}

Ammer, A., \& Bertel, K. (2011). \#Socialtagging: Defining its Role in the Academic Library. School of Information Student Research Journal, 1(2). https://doi.org/10.31979/2575-2499.010203 Retrieved from https://scholarworks.sjsu.edu/ischoolsrj/vol1/iss2/3

This article is brought to you by the open access Journals at SJSU ScholarWorks. It has been accepted for inclusion in School of Information Student Research Journal by an authorized administrator of SJSU ScholarWorks. For more information, please contact scholarworks@sjsu.edu. 


\section{\#Socialtagging: Defining its Role in the Academic Library}

\section{Acknowledgements}

The authors would like to thank their professors, Valerie Nesset and Brenda L. Battleson, of the Department of Library and Information Studies at the University at Buffalo, The State University of New York, for their guidance and support.

\section{About Author}

Annalise Ammer is currently pursuing her MLS at the University at Buffalo, The State University of New York, with an expected graduation date of May 2012. Her research interests include digitization of artifacts and texts, user interface design, and virtual libraries. Upon graduating she hopes to find a position in a digital library setting in either an academic or special library.

Katherine Bertel is a current MLS student at the University at Buffalo, The State University of New York, with an expected graduation date of May 2012. Her research interests include user-centered library instruction, modern information retrieval, and emerging technologies. She hopes to find a position in an academic library. 
\#Socialtagging: Defining its Role in the Academic Library

The past decade has brought about many changes in the way people search for and organize information, especially within the online environment. The Internet dramatically increased the amount of information available to the public, leading to an increase in the need for meaningful organization. Recent developments in Internet technologies have led to the creation of Web 2.0 tools, particularly social tagging websites. Such tools rely heavily upon user participation: Social tagging depends on users to create and apply tags to documents, images, video clips, and other resources. Social tagging has allowed for the creation of more access points to more resources in a way that is easier for more users to employ when compared to controlled vocabularies and subject headings. This idea is supported by many researchers within the information-seeking community. Kakali and Papatheodorou (2010) stated that "community engagement in social tagging creates a common vocabulary, reflecting, usually, a personalized conceptual model of the world, rather than a hierarchical model of knowledge categorization" (p. 192). However, there are conflicting opinions as to whether social tagging would be a good addition to traditional indexing and knowledge management methodologies.

Numerous researchers within the library science field have found that social tagging allows for more flexibility within an information retrieval system because "user assigned tags could cover aspects that are not available in a knowledge organization system, especially when it comes to new concepts; as such, they could help update the knowledge organization system" (Matthews et al., 2010, p. 448). At the same time, other scholars believe there is too much flexibility with social tagging and not enough control over the vocabulary (Hayman \& Lothian, 2007; Lu, Park, \& Hu, 2010). While these are both valid viewpoints, social tagging is here to stay. Now is the time for librarians to incorporate this widely used tool into the academic library catalog, allowing for a more participatory librarianship. Social tagging is not meant to replace subject headings or controlled vocabularies; instead, tags can be used concurrently to improve access according to changing user needs in the digital age. By using social tagging to complement subject headings, the main mission of a library to provide the highest level of access to the most users can be achieved.

\section{Review of the Literature}

\section{Today's Information Seeker and User}

The prominence of the Internet and digital technologies in academic life has changed the way students use and search for information. Library collections have 
multiplied in size as more collections are digitized and added to institutional repositories, and resources are being shifted from amassing collections of physical objects to providing access to information. Digitization of information has increased the need for new ways of accessing information. Traditionally, access to library collections has been provided through a centralized location, classified by controlled languages (subject indexing) developed by information professionals.

Now, users can access information anywhere there is an Internet connection, and the organization and control of information is not limited to information professionals or libraries. Due to the amount of information available on the Web and the sheer size of digital collections, it has proven difficult to use controlled vocabularies when indexing digital information (Kipp, 2010; Redden, 2010). Additionally, "it is often difficult for library users to formulate a proper query using specific keywords assigned to different fields of desired library catalogue records" (Lu, Park, \& Hu, 2010, p. 768). Therefore, students need a new way of accessing information that coincides with their usage behavior and expectations. With the changing technological landscape, a new set of expectations has been created through the development of Internet-based technologies. The development and proliferation of Web 2.0 tools has changed the way users are able to interact with information.

The term Web 2.0 applies to a set of characteristics found in Web-based tools and practices that encourage more user interaction and participation (Conole \& Alevizou, 2010). Student users of information not only want to interact and participate in an online environment, they have come to expect it (Steele, 2009). The Internet is now a collaborative and interactive environment rather than a static one (Redden, 2010). In this new environment, more users than ever before are both consumers and contributors of information.

The range of published content has expanded to include blogs, wikis, and social networks. The change in the production of information has caused a shift toward a more participatory and user-oriented library in which content comes from both librarians and users. The ability for users to contribute to the information process is an especially important aspect of librarianship in the digital era. Librarians once dominated the maintenance of taxonomies, controlled vocabularies, and other information classification structures. Web 2.0 technologies have made this process potentially available to all. Now, with options like social tagging, the task (classification) is not reserved for librarians (Hayman \& Lothian, 2007; Ivey, 2009). Users view classification as a personal process closely attached to their work (Lu et al., 2010). Therefore, students in the academic library need a personal space where they can manage and organize their work. It is also important that students understand the differences in the types of tags used, especially in regards to pure social tagging versus tags used by the entire academic community. 


\section{Defining Social Tagging}

When discussing the implications of social tagging, we must first define what social tagging is. Due to its relatively recent emergence onto the information organization and retrieval scene, there are many different definitions, some more accurate than others. Voss (2007) defines social tags as user-generated, reusable annotations, and subject search terms that are applied to any media type. This definition of social tagging is frequently used when compared with manual indexing. With social tagging, "the basic principle is that end users do subject indexing instead of experts only, and the assigned tags are being sown immediately on the web" (Voss, 2007, p. 2). In addition, many interchangeable terms exist alongside the many definitions of social tagging: collaborative tagging, social classification, social indexing, folksonomy, and social annotation.

Tags can be applied to almost any information-containing item, such as pictures, videos, websites, articles, documents, books, blogs, or music. Depending on the application used, oftentimes there are no limits to the number of tags that can be applied to an item. Web 2.0 services either provide a separate window to add tags, or give users the option of denoting tags through the use of hashtags (words or phrases prefixed with a hash symbol [\#]; the title of this paper is a nod to this tagging method). Tags are then displayed in a sidebar so users can easily use, add, delete, or modify tags. The social aspect of tags refers to the community effort to tag documents, and all tags applied to a document can be viewed by other social taggers in the community. Tag clouds allow members of an online community to see what others have tagged, as well as the popularity of a tag. Unlike traditional subject classification and subject headings, social tagging is not the work of experts. Instead, social tagging is performed by the end user, creating a whole new set of information retrieval concepts and outcomes. Tagging cannot be forced upon the user-instead, the user must want to participate.

Types of tagging. The word tagging is an umbrella term for a whole classification of tags available to use. Steele (2009) distinguishes between two main types of tags-broad tags and narrow tags-and their seven sub-types. Broad tags are for use by the community; they are broad by nature, making them good for general topics and ideas. Narrow tags, in contrast, are used primarily for personal information organization and retrieval. The seven sub-types are descriptive, resource, ownership/author, opinion, self-reference, task-organizing, and play tags. Descriptive tags tend to be more controlled and similar to subject headings. Resource tags perform a basic function: They describe what the item being tagged is. Ownership tags state who wrote or published the item. Opinion tags state the social taggers' opinions of the document, such as "good," "funny," or "bad." Two similar, private tag sub-types—self-reference and task-organizing 
tags - are largely created only for individual taggers to help organize and control their information. Finally, play tags are for entertainment purposes (Steele, 2009). By understanding the variety of tag types, researchers can better analyze what tags are used more frequently and how particular tags can be applied in an academic or traditional library setting.

\section{Semiotics Approach to Tagging}

Social tagging serves as a modern example of semiotic theory. The field of semiotics is concerned with everything that can be viewed as a sign (Eco, 1984). Signs are not limited to the act of speech or language, but are concerned with anything that can be used to represent something else. Tags applied to an item are symbols that represent the content of the item. Tagging permits the use of numerous symbols (tags) to be applied to any given item. By allowing multiple users to apply their own tags to a single document, that document takes on a greater level of meaning and accessibility. Social tagging allows multiple symbols to be assigned to a single document from multiple viewpoints, which makes the tagged items more accessible to all. Markines et al. (2009) support the concept of creating a larger base of tags for increased accessibility as "they allow us to extend the assessment of what a page is about from content analysis algorithms to the collective "wisdom of the crowd"' (p. 1). Unlike traditional indexing, tagging provides a more creative and flexible approach for applying appropriate terms, leading to a more user-oriented environment. The meaning of a sign manifests in the interpretation created by its users, the most distinctive and innovative component of Peirce's Sign Theory (Atkin, 2010).

Much of the research available on social tagging and semiotics refers to the triadic sign theory developed by the American philosopher, Charles Sanders Peirce (Atkin, 2010; Huang \& Chuang, 2009). The triadic sign theory consists of three elements: representation, object, and interpretant. Huang and Chuang (2009) apply this theory to the process of social tagging in their own research; they "regard social tagging as a sign based on its triadic relation, that is: Representation (social tagging), Object (online communication) and Interpretation (Peircean semiotics)" (p. 341). Their research compares social tagging to the theory of Peircean semiotics, particularly the 10 classes of signs, and Huang and Chuang determine how to improve online communication through improved tagging techniques. They believe that social tagging is the representation; in other words, the text applied to the document as the tag is the sign itself. The object is the entity to which the tag is being applied: website, image, document, and so on. The interpretant portion is the user's own interpretation of the tag that has been applied (see Figure 1). 


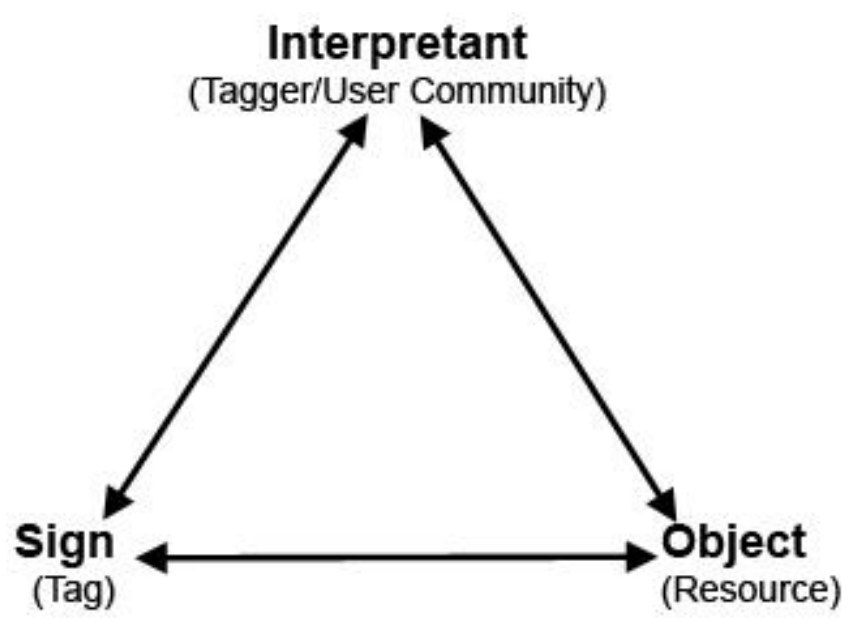

Figure 1. Peirce's triad of semiotics applied to social tagging. Image adapted from Huang \& Chuang (2009).

Within this structure, anyone can interpret a sign as he or she sees fit. Given human nature, not everyone will interpret a sign the same way; therein lies the issue. Tags can be interpreted incorrectly for a variety of reasons such as spelling variations, homonyms, and slang. To make tags more widely understood and accurate, Huang and Chuang (2009) recommend organizing tags based on the Peircean 10 classes of signs, through which tags will still be largely creative, but will contain a theoretical structure that makes them more effective and enables them to improve online communication. Huang and Chuang (2009) "look to semiotics for the concepts and general principles that are relevant and significant," and their research "identifies 10 classes of social tagging to offer a semiotic solution to the vagueness and ambiguity of tagging in the online communication process" (p. 355). As Huang and Chuang demonstrate, applying semiotic theory to social tagging can eliminate the issues that come along with tagging, particularly synonyms, polysemes, and inconsistency.

Social tagging and semiotics have also been analyzed by Voss (2007) in reference to subject indexing. He believes that social tagging has helped to rekindle manual indexing in a world that has become largely based on full-text searching. Voss (2007) states that "subject indexing involves two steps: conceptual analysis and translation" (p. 4). Voss claims that social tagging also includes these two steps, thus strengthening the comparison to manual indexing. Conceptual analysis requires a user to interpret a document and determine its essence. 
When a social tagger views or reads an item, he or she establishes what the document is about, declaring its aboutness. Unlike manual indexing, which is usually performed by one person, social tagging allows multiple taggers to index items. Research has shown that "conceptual analysis heavily depends on the needs and interest of users that a resource is tagged for - different people can be interested in different aspects" (Voss, 2007, p. 4). Multiple interpretations of the document can be determined by each individual tagger, thus increasing the number of access points for the document. Translation is the act of applying a tag appropriate to what the tagger or indexer determines during the conceptual analysis step, and problems usually occur during the translation step due to the participation of multiple social taggers. When more than one person is tagging an item, it becomes very difficult to ensure consistency among the tags.

\section{Tagging in the Academic Library}

In the past, the library has been a relatively static environment. Interaction from library users has generally been limited to the search and retrieval of documents from the catalog. Social tagging provides users and librarians a new way of interacting with the catalog. Experts are no longer the only ones organizing and managing information. Many librarians fear relinquishing their control for a system that is inherently somewhat wild (has no authority control). However, social tagging is not meant to replace subject headings and controlled vocabularies-rather, it is intended to complement them. This symbiosis between the knowledge organization system and tagging can be achieved in multiple ways.

Where social tagging is weak or flawed (such as having a large variety of terms), subject headings are strong (such as providing a rigid structure), and vice versa. In this way, tagging can be used to start the search because users can find tags that are based on natural language; then, subject headings can be used in the retrieval of related documents. Suggestions for tags can also come from the controlled vocabulary or knowledge organization system, providing users with a more precise language and thereby eliminating some problems commonly associated with tagging. With the implementation of social tagging, the catalog is a collaborative space where different viewpoints are represented and everyone can participate. With the ability to obtain information interactively, users may find the catalog less confusing and less difficult to navigate. As a result, the library becomes more welcoming to a greater number of users (Steele, 2009).

Social tagging relies on participation, which is especially important in the academic library. A seemingly intuitive statement, but if not taken into consideration, the time, money, and effort exerted to implement a social tagging system can go to waste. If users do not participate, then the system fails. When 
librarians incorporate social tagging into the library, they must make tagging easy, useful, and fun. As Steele (2009) puts it:

The library has to make sure the tagging system helps people manage their information well; otherwise it could become just another "information closet" like bookmarks. Encouraging collaboration, self-expression, and play is another way to ensure patron participation. In other words, make tagging fun and useful, not just a chore. (p. 76)

Technological tools such as social tagging can enable librarians to facilitate convenient access to library resources, provide access to services or resources unavailable before, or provide support services when help is needed. However, if the proposed tool is not easy to use, it can cause many problems for the user: Users can become frustrated or intimidated; complex technologies can impede interaction and create distance between users and the library (Walker, Craig-Lees, Hecker, \& Francis, 2002). Not only should the proposed tool be easy to use, but students should also be invited to participate, thus enabling them to express their unique viewpoints. These issues must be taken into consideration when assessing the potential benefit of a technological tool.

Examples of implementation. One of the most successful examples of social tagging integrated in an academic library has been the University of Pennsylvania PennTags system (University of Pennsylvania, 2004). PennTags is a locally developed tagging software program created by the University of Pennsylvania. Members of the University of Pennsylvania community can tag or save cataloged books, journals, articles, webpages, images, and query results. The PennTags system caters to users who are comfortable with traditional search methods and those seeking a new way of interacting with the catalog. Features of the PennTags website include a tag cloud that visualizes the size of tags according to popularity (tags must be used at least 110 times before they are displayed in the tag cloud); a section for recently tagged items; and a quick-access pane for tag groups and projects. According to statistical data, 27 bookmarks (on average) are posted each day and every post is assigned four tags (Lu et al., 2010; Steele, 2009). Developing an in-house system, however, is not the only option available to librarians considering the possibility of implementing tagging.

Librarians also have the option of using external websites, such as LibraryThing (http://www.librarything.com/), for tagging documents. LibraryThing is a popular Web 2.0 tool with a social dimension that allows users to share and view other user profiles, as individual users catalog their books. One negative aspect of using externally hosted websites in the academic library setting is that students are required to create a profile in order to participate. As simple as 
this can be, it can be a big turnoff for many students because this requires more effort from the user to maintain and update stored information, manage passwords or usernames, use different accounts on multiple websites, and perform multiple steps to get to the information needed (Redden, 2010). When libraries have opted to use external websites for tagging, this has proven to be less sustainable compared to locally developed systems. When a college or university decides to develop a locally developed system, librarians and other library staff are often more invested in its implementation and success. An externally hosted system, on the other hand, can easily be forgotten or neglected. For example, Ohio State University's use of LibraryThing was chronicled by Steele (2009), but their profile no longer exists. This indicates that adoption of Web 2.0 tools must be carefully thought out and planned prior to implementation.

In order for the adoption of external websites to be successful, librarians must maintain and promote their usage. Before a library adopts the use of any Web 2.0 tool, there should be a plan to regulate the person(s) in charge of maintaining the library's profile and presence. One added benefit to using external tagging websites is that the users can take advantage of the expansive vocabularies from these websites. Additionally, it costs the library very little to use and maintain an external website. If the external website proves to be a success, it could lead to the in-house creation of a tagging system tailored to that particular library and its users.

\section{Implications to Knowledge Management}

\section{Controlled Languages}

Subject headings are a vital component of the academic library. They provide a structure that allows information to be organized in a consistent and precise manner. Subject headings such as the Library of Congress Subject Headings (LCSH) are based on a hierarchy with headings and subheadings. This structure eliminates sense ambiguity between homographs, synonyms, and polysemes by placing the term within a context. A word is defined by the company it keeps, thereby providing a clear and precise meaning for the user. Subject headings and controlled vocabularies are often created according to standards, such as the National Information Standards Organization (NISO) or American National Standards Institute (ANSI). Adherence to such standards eliminates problems such as misspellings and plural word forms, while maintaining consistency.

In addition to quality control, librarians take issue with the potentially short life span of social tagging. Subject headings have proven to have greater longevity than social tagging. According to Matthews et al. (2010), 
Libraries have cataloged millions of volumes using the LCSH, and it would take years to tag all the items in the catalog. Some materials could probably never be tagged. Therefore, a tag search is going to come up with only the most recent or popular information. (p. 72)

The proven longevity of subject headings has caused many librarians to question the staying power of social tagging. Many librarians believe social tagging is just another fad and believe their efforts to implement tagging would be irrelevant a few years from now. However, there is a dire need to update the way users are able to access information.

Many aspects of subject headings can actually impede rather than improve a user's access to information. Information is increasing at an alarming rate and concepts are constantly changing. Due to their size, subject heading systems react slowly to new concepts and changes in meaning. Subject headings such as LCSH have evolved to accommodate contemporary terms, but they can never be completely up to date. Although updates have been made, terms that are either archaic or deviate from common usage can still be found. While the subject headings are intended to be helpful, they sometimes use unpopular terms that impede the user's ability to locate and use the "correct" term.

It is often very difficult for non-experts to use subject headings for formulating queries because they are created by expert users. Trant (2006), assessing the relationship of the folksonomy (tags created by a community of users) to the professionally created museum-controlled vocabulary, revealed that $86 \%$ of user-generated tags were not present in the museum's controlled vocabulary. This study indicates that social tagging provides a significantly different vocabulary not available from expert-generated systems of knowledge organization (Lu et al., 2010). According to Cory Doctorow, a Creative Commons activist and author, "requiring everyone to use the same vocabulary to describe their material denudes the cognitive landscape, enforces homogeneity in ideas. And that's just not right" (as quoted in Steele, 2009, p. 72). No single term, however well-crafted and chosen, can cover all of a user's query attempts. Our language and our experiences are varied and colorful. Users should not be forced to use only one viewpoint when searching for information. This limits the ways users are able to think about and articulate their topic. A system should support the vocabulary of all users to best serve the library's mission of providing the most access to the most users.

\section{Social Tagging}

Compared to traditional indexing techniques, social tagging has many features that improve the overall experience for the end user. One of the main features that 
draw users to tagging is that the tags can be written in the user's natural language. The vocabulary that they use to tag has more meaning to users than do traditional subject headings, therefore increasing their overall understanding of the document at hand. Spiteri (2007) writes that "an attractive feature of folksonomies is their inclusiveness; they reflect the vocabulary of the users, regardless of viewpoint, background, bias, and so forth" (p. 14). The user's ability to interact with the information removes the barrier between the user and the item. Instead of being presented with static information, the user becomes actively involved and can organize information as he or she sees fit. The environment with which users interact is changing: "The user is in control. The patrons are changing, and are expecting to be able to participate and interact online" (Steele, 2009). Social tagging encourages participation and fosters a sense of community among the taggers. Before social tagging, information retrieval was a solitary act. Now people work together as groups to help organize and disseminate information, making it more accessible for everyone.

Although there are many positive aspects to social tagging there are drawbacks as well. One of the most discussed issues in research is the lack of authority control in social tagging. Kakali and Papatheodorou (2010) point out: "In contrast to traditional classification systems and thesauri, there is no authority control, nor are there selection criteria and instructions for tag generation, and as a result many synonymous tags are generated" (p. 192). Issues that occur with tagging include ambiguity, polysemy, and synonymy. One word can have many different meanings, such as the word present, which can mean "current," "a gift," "to show," or "to be somewhere." A tag can be too specific or too general depending on who is utilizing the document and the tag.

Traditionally, tagging systems do not provide guidelines to users concerning the form of tags, thus leading to non-conformity (Spiteri, 2007). When tags are not clear or concise, their purpose of communicating information and helping others find information is diminished. Huang and Chuang (2009) state that "confusion in social tagging, seen from the perspective of online communication, occurs because of different interpretations of what tags are meant to represent. More specifically, the difficulty lies in the interpretation along technical and social dimensions" (p. 341). A potential solution to this problem would be the addition of some form of authority control over the tags.

To find out whether authority control or tag suggestions would improve tag quality, a study described by Matthews et. al. (2010) provided participants with documents that were to be tagged. The study involved 10 participants who were considered active (i.e., having published and deposited a number of papers to the institutional repository, ePubs) in a single field of study: computer science and information technology. This particular field uses an easily available controlled vocabulary: the ACM Computing Classification Scheme. The study 
was able to compare this widely-used vocabulary against the vocabulary of the user (Matthews et al., 2010). Some of the documents to be tagged by the subjects had suggested tags (controlled vocabulary terms), while other documents had no suggestions and were open to interpretation. After the procedure was finished, the general conclusion was that "choosing terms from a controlled vocabulary was a 'good thing' and better than choosing their own terms," and that "usability would be greatly enhanced by providing some automatic assistance in suggesting tags" (Matthews et al., 2010, p. 460).

Overall, it seems that suggested tags from a controlled vocabulary would be a potential benefit to the user, but they cannot be the only option available for the social tagger. If tags only came from the controlled vocabulary, that would defeat the purpose of tagging. Steele (2009) makes this balance between authority control and flexibility clear in his research, stating that "the users must be allowed to create their own tags, and not forced to choose from a selection. While the system can offer suggestions, the option to add their own still must exist" (p. 69). Once again, this supports the idea that tags need to strike a balance between rigidity and flexibility in order to be successfully implemented.

Social tagging and manual indexing. There is much discussion as to whether social tagging helps improve indexing, or whether it has any effect on indexing at all. Some researchers believe that social tagging is helping to bring about a reform in manual indexing. Over the past decade, manual indexing has decreased in popularity because of the high cost and the extensive time its creation requires. Until social tags became popular, almost all online searching was performed through full-text searching. Both indexing and social tagging allow more specific and descriptive information seeking than does full-text searching.

To determine which method (indexing or social tagging) would be more cost efficient and beneficial, a document must be evaluated. For a static document such as a book, indexing makes more sense. Documents that are not as staticsuch as blog posts, websites, photos, podcasts, videos, or comments - are better suited for tagging. For these dynamic items that naturally and continually evolve, tags will be more likely to always reflect its content due to the flexible and everchanging nature of tags. Furthermore, tags "hold the promise of reducing indexing costs by drawing end-users into contributing, adding value as part of their interaction with formation services" (Matthews et al., 2010, p. 448). Overall, manual indexing and social tagging are similar in nature. They complement one another while maintaining separate and equally important identities and uses. 


\section{Discussion}

\section{Assessment}

Due to its increased popularity over the past five years, the influence that social tagging has on today's information-seeking user can no longer be ignored. Social tagging is here to stay, so information professionals should continue to explore social tagging to the fullest extent possible. This review of the research available on social tagging and its usability in traditional libraries indicates that tagging can never entirely replace traditional indexing and subject headings. Instead, a system that incorporates both traditional methods and tagging in a cohesive manner seems to be in order. Today's users expect to be able to interact with the information available, and tagging has created an environment that allows them to do so. Social tagging can be the bridge between modern users and libraries, as "social bookmarking and tagging tools help librarians bridge the gap between the library's need to offer authoritative, well organized information and their patrons' web experience" (Rethlefsen, 2007, p. 26). Although the flexibility of tagging is a positive characteristic, it must be partially controlled in order to successfully implement a social tagging system in a traditional library setting. Tag suggestions or an optional authority control would greatly improve the quality of tags, thus allowing tagging to be consistent, effective, and efficient in an academic or traditional library setting.

\section{Suggestions for a Methodology to Control Tags}

Social tagging is a tool with which many students are already familiar; therefore, any system a library decides to adopt should mimic features of popular tagging systems. Features of popular tagging websites include tag ranking, recommendations, and reviews. Popular e-commerce websites, such as Amazon.com, serve as successful models that include many of these features. Based on past tagging behavior, the tagging system can recommend related tags or provide suggestions for tags based on users who have tagged similarly or from the knowledge organization system. Automated features such as these can improve the tagging quality and indexing exhaustivity.

Another methodology for controlling tags is to limit the modification of tags to the academic community. This can be further reduced to creating specialty or niche user groups, according to departments or majors, who are in charge of managing the tags of that specialty. Marlow and Miller (2011) state, "the Expert User title would require that they have some proficiency with the subject matter or credentials in order to accurately tag the image" (p. 5). Therefore, the judgment made by the expert user on the relevancy and usefulness of a tag could be valued. 
According to a study performed by Nichols and Mellinger of Oregon State University, it was discovered that undergraduate students search for information according to course assignments and projects whereas graduate students and faculty search according to subject (Redden, 2010). Providing the ability for users to create groups according to subject helps improve the access to and retrieval of information more relevant to that particular user. Additionally, by allowing niche users to control tags, users more easily engage with the library. Users would be considered experts, providing them with the motivation to participate because they have a specific role that contributes to the betterment of the library for the good of the community. These expert users would also have a special interest in ensuring that tags are used properly and documents are tagged appropriately, and they could help monitor and correct the use of personal tags.

Personal tags can become problematic when "many users use tags only to characterize their own documents, and not to help the community" (Matthews et al. 2010, p. 448). Providing users with the option to tag items for their own personal use or community use would help reduce the prevalence of self-reference tags such as "read," "unread," or "owned." Because users like to attach personal resource management while tagging, this would provide users the option to use these tags but they would not be added to the community (Lu et al., 2010).

\section{Conclusions and Future Research}

Social tagging is a burgeoning topic of research in the library science field. To date, its implementation in the academic library has been slow. Suggestions for further research on social tagging in the academic library setting include:

- further implementation of social tagging and subject headings being used concurrently;

- studies of user interaction with a controlled language and/or suggestionbased tagging vocabulary system;

- investigation into whether users find tags or subject headings more useful when searching; and

- studies involving participants in fields outside of library science to better represent typical users.

When used correctly, social tagging can be an excellent tool for improving access to a library's catalog. Academic library websites should incorporate "an efficient, flexible, and user-oriented interface, build a virtual space that facilitates rich user experiences, engage users and encourage collective intelligence and support content sharing and nurture online communities" (Redden, 2010, p. 224). By incorporating social tagging into the academic library, users have the ability to 
become more engaged with the creation and dissemination of information through personal or community-based tagging environments. These environments also allow many viewpoints to be represented equally. The differing viewpoints of an increasingly diverse demographic are better represented in a system that reflects each user's unique cultural perspective, allowing the aboutness of an object to be determined by many people, and such a system can reflect the perspective of all users. Social tagging is a tool that meets these requirements. For successful implementation, however, information professionals must use social tagging with a carefully balanced level of authority control that allows for flexibility while minimizing inaccuracies.

\section{References}

Atkin, A. (2010). Peirce's theory of signs. In E. Zalta (Ed.), Stanford encyclopedia of philosophy (Winter ed.) Retrieved from http://plato.stanford.edu/archives/win2010/entries/peirce-semiotics

Conole, G., \& Alevizou, P. (2010). A literature review of the use of Web 2.0 tools in higher education. Report commissioned by the Higher Education Academy. Milton Keynes, UK: Open University. Retrieved from http://oro.open.ac.uk/23154

Eco, U. (1984). Semiotics and the philosophy of language. Bloomington, IN: Indiana University Press.

Hayman, S., \& Lothian, N. (2007, August). Taxonomy directed folksonomies: Integrating user tagging and controlled vocabularies for Australian education networks. Paper presented at World Library and Information Congress / 73rd Annual IFLA General Conference and Council, Durban, South Africa.

Huang, A., \& Chuang, T. (2009). Social tagging, online communication, and Peircean semiotics: A conceptual framework. Journal of Information Science, 35(3), 340-357. doi:10.1177/0165551508099606

Ivey, R. (2009). Perceptions of the future of cataloging: Is the sky really falling? Cataloging and Classification Quarterly, 47(5), 464-482. doi:10.1080/01639370902832645 
Kakali, C., \& Papatheodorou, C. (2010). Exploitation of folksonomies in subject analysis. Library and Information Science Research, 32(3), 192-202.

Kipp, M.E., \& Campbell, D.G. (2010). Searching with tags: Do tags help users find things?. Knowledge Organization, 37(4).

Lu, C., Park, J., \& Hu, X. (2010). User tags versus expert-assigned subject terms: A comparison of LibraryThing tags and Library of Congress Subject Headings. Journal of Information Science, 36(6), 763-779. doi:10.1177/0165551510386173

Markines, B., Cattuto, C., Menczer, F., Benz, D., Hoto, A., \& Stumme, G. (2009, April). Evaluating similarity measures for emergent semantics of social tagging. Paper presented at 18th International World Wide Web Conference, Madrid, Spain. Retrieved from http://www2009.eprints.org/65/1/p641.pdf

Marlow, L. L., \& Miller, A. (2011, April). A picture is worth a thousand words: The perplexing problem of indexing images. Paper presented at University at Buffalo Graduate School of Education Student Research Symposium, Buffalo, NY.

Matthews, B., Jones, C., Puzon, B., Moon, J., Tudhope, D., \& Golub, K. (2010). An evaluation of enhancing social tagging with a knowledge organization system. Aslib Proceedings, 62(4/5), 447-465. doi:10.1108/00012531011074690

University of Pennsylvania. (2004, October). PennTags. Retrieved from http://tags.library.upenn.edu

Redden, C. (2010). Social bookmarking in academic libraries: Trends and applications. The Journal of Academic Librarianship, 36(3), 219-227. doi:10.1016/j.acalib.2010.03.004

Rethlefsen, M. (2007). Tags help make libraries Del.icio.us. Library Journal, 132(15), 26-28.

Spiteri, L. (2007). Structure and form of folksonomy tags: The road to the public library catalog. Information Technology and Libraries, 26(3), 13-25. 
Steele, T. (2009). The new cooperative cataloging. Library Hi Tech, 27(1), 68-77. doi:10.1108/07378830910942928

Trant, J. (2006). Exploring the potential for social tagging and folksonomy in art museums: Proof of concept. New Review of Hypermedia and Multimedia, 12(1), 83-105. doi:10.1080/13614560600802940

Voss, J. (2007). Tagging, folksonomy and co-renaissance of manual indexing. Proceedings of the International Symposium of Information Science, Cologne, Germany. Retrieved from http://arxiv.org/PS_cache/cs/pdf/0701/0701072v2.pdf

Walker, R., Craig-Lees, M., Hecker, R., \& Francis, H. (2002). Technologyenabled service delivery: An investigation of reasons affecting customer adoption and rejection. International Journal of Service Industry Management, 13(1), 91-106. doi:10.1108/09564230210421173 